\title{
DETERMINACIÓN DE LA CURVA DE DILUCIÓN DE NITRÓGENO EN DIFERENTES FASES FENOLÓGICAS DEL GIRASOL
}

\author{
Determination of the nitrogen dilution curve in different phenological \\ phases of sunflower
}

\author{
Gladis A. de Caram¹, Patricia Angeloni ${ }^{1}$ y Juan Prause ${ }^{1 *}$
}

\begin{abstract}
A B S T R A C T
The concentration of nitrogen which allows a crop to reach its maximum growth rate is called critical concentration. This concentration varies according to the aerial biomass considered, as when this increases, there is a dilution effect of the nitrogen in the plant. The aim of this work was to characterize the nutritional state of foliar nitrogen in two sunflower hybrids (Helianthus annuus L.), at different phenological phases, using the nitrogen dilution curve in total aerial dry matter. The treatment was applied to two hybrids, GUARANI DC and DKSOL 4040, sown in four furrows by plot, making three replicates per each hybrid. The sampling was made taking three plants per treatment for each replicate, and in four phenological phases: V6; V10; R1; R5.5 and R6. Dry matter, nitrogen content and foliar nitrogen were determined by the semimicroKjeldahl method (B). By using the nitrogen dilution curve it was detected that the content of nitrogen in both hybrids was higher than the required quantity for the maximum growth rate till the flowering, and that the nitrogen values obtained in R1 and R6 were under the curve of dilution. This methodology is not practical to judge the nitrogenous state of the crop in the stages after flowering, because the composition of the dry matter changes after this phase and the oil synthesis phase begins.
\end{abstract}

Key words: nitrogen dilution, phenological phases, sunflower, Helianthus annuus.

\section{R E S U M E N}

La concentración de nitrógeno que le permite al cultivo alcanzar la tasa de crecimiento máxima se denomina concentración crítica; estas concentraciones varían de acuerdo a la biomasa aérea considerada, ya que a medida que ésta aumenta se produce un efecto de dilución del nitrógeno en la planta. El objetivo del trabajo fue caracterizar el estado nutricional en nitrógeno foliar de dos híbridos de girasol (Helianthus annuus L.), en distintas fases fenológicas, empleando la curva de dilución del nitrógeno en la materia seca aérea total. Los tratamientos fueron dos híbridos, GUARANI DC y DKSOL 4040, que se sembraron a razón de cuatro surcos por parcela, realizándose tres repeticiones por híbrido. El muestreo se realizó tomando tres plantas por tratamiento para cada repetición, en cinco fases fenológicas: V6; V10; R1; R5.5 y R6. Se determinaron materia seca, contenido de nitrógeno y nitrógeno foliar por el método semimicroKjeldahl. Utilizando la curva de dilución del nitrógeno se detectó que el contenido de nitrógeno en ambos híbridos fue superior a la cantidad necesaria para el cultivo hasta la floración y que los valores de nitrógeno obtenidos en R1 y R6, estuvieron por debajo de la curva de dilución. Esta metodología no es utilizable para juzgar el estatus de $\mathrm{N}$ del cultivo en estadios posteriores a floración, ya que después de dicha fase, cambia la composición de la materia seca, dando comienzo a la etapa de la síntesis de aceite.

Palabras clave: dilución de nitrógeno, fases fenológicas, girasol, Helianthus annuus.

\footnotetext{
${ }^{1}$ Universidad Nacional del Nordeste, Facultad de Ciencias Agrarias, Cátedra de Agroclimatología, Sargento Cabral 2131, (3400) Corrientes, Argentina. E-mail: prause@agr.unne.edu.ar *Autor para correspondencia. Recibido: 13 de diciembre de 2005. Aceptado: 11 de mayo de 2006.
} 


\section{INTRODUCCIÓN}

El girasol (Helianthus annuus L.) es un cultivo que puede desarrollarse satisfactoriamente en diversos ambientes de la República Argentina, desde el sudeste de la Región Pampeana ( $\left.39^{\circ} \mathrm{S}\right)$ hasta la Región Chaqueña $\left(26^{\circ} \mathrm{S}\right)$ (Satorre et al., 2004). La región noreste ha contribuido notoriamente a la expansión del mismo en los últimos años, no obstante, se dispone de escasa información sobre el cultivo del girasol en la Provincia del Chaco, Argentina, específicamente de conocimientos básicos que permitan optimizar su manejo. Es una planta anual y herbácea, cuya importancia económica radica fundamentalmente en su uso como oleaginosa, por su alto contenido de aceite en las semillas, tanto para consumo humano como también en la industria oleoquímica. El aceite de girasol es de alta calidad comestible, debido a su alto contenido de ácido linoleico y vitamina $\mathrm{E}$, lo que le confiere un gran valor nutritivo.

El patrón de crecimiento y desarrollo fenológico de esta oleaginosa está regulado por la disponibilidad de agua y nutrientes en interacción con los factores ambientales (Andrade et al., 2002). El rendimiento alcanzado por el cultivo en la mayoría de los casos se debe a la optimización de variables, como fecha de siembra, disponibilidad de agua y nutrientes, temperatura, precipitaciones, etc. La tasa de producción de materia seca está determinada por la cantidad de radiación interceptada, la cantidad de agua disponible y por la disponibilidad de nutrientes (Connor y Sadrás, 1992; Andrade, 1995).

Entre los nutrientes, el nitrógeno es el que en mayor medida limita la producción de girasol en la Argentina, de ahí la importancia de conocer los requerimientos nutricionales y realizar diagnósticos de deficiencias (Díaz-Zorita, 2002). El nitrógeno juega un papel clave en la estructura de las proteínas y ácidos nucleicos y puede considerarse entonces, junto con el carbono, como uno de los elementos centrales de la vida (Loomis y Connor, 2002). El girasol requiere un alto nivel de nutrición con $\mathrm{N}$ durante el crecimiento vegetativo, para la obtención de elevados rendimientos de grano y proteína, afectando la producción de materia grasa, ya que la meta del cultivo es mayor rendimiento en grano con mayor porcentaje de aceite (Aguirrezábal et al., 1996). A pesar de ello, existe limitada información sobre la respuesta del girasol a la fertilización ni- trogenada, en comparación con otros cultivos. En la mayoría de las situaciones el porcentaje de aceite tiende a decrecer a medida que aumenta el porcentaje de proteínas, debido a incrementos en la disponibilidad de nitrógeno (Díaz-Zorita, 1997; Sadrás et al., 2002; Satorre et al., 2004). Rufo et al. (2003) encontraron que la fertilización con nitrógeno en girasol, incrementa la producción de granos y aceite por hectárea, pero no tiene efecto sobre la concentración de aceite en el grano.

Al generalizarse el uso de materiales genéticos de alto potencial de producción, los que incrementaron significativamente sus rendimientos promedios y por lo tanto los requerimientos nutricionales de los mismos (Andrade et al., 2002), se hace necesario el estudio de estos requerimientos. Es sabido que el $\mathrm{N}$ juega un rol importante, ya sea directa $\mathrm{o}$ indirectamente, en procesos como crecimiento $\mathrm{y}$ senescencia foliar y la determinación de los componentes del rendimiento del girasol, los que se definen en diferentes etapas del cultivo (Merrien, 1992). Este macronutriente es el que genera la cobertura a través de la expansión foliar, por ello deficiencias durante la etapa vegetativa temprana reducen el número de hojas o sea el crecimiento y el rendimiento, mientras que deficiencias posteriores afectarán el número y peso de los frutos (Connors y Sadrás, 1992). La mayor absorción de $\mathrm{N}$ y de $\mathrm{P}$ se concentra en los 30 a 35 días previos a la floración, durante la etapa de crecimiento rápido del cultivo, la cual cesa cuando comienza la floración. González Montaner et al. (1995) encontraron que el girasol absorbe entre 40 y $60 \mathrm{~kg}$ de $\mathrm{N}$ por tonelada de aquenio, y que la respuesta del girasol a la fertilización es errática y condicionada por diversos factores (Satorre et al. 2004), no encontrando relaciones consistentes entre absorción y rendimiento, debido a la interacción entre factores que puedan afectar el estado fisiológico de la planta, tales como radiación, temperatura, disponibilidad de agua, compatibilidad genética, ocurrencia de enfermedades, etc.

La información disponible sobre herramientas de diagnóstico de necesidades de fertilización nitrogenada es muy variada. Una de ellas considera el análisis en plantas durante el estado de desarrollo vegetativo del cultivo, estimando el contenido de $\mathrm{N}$ por encima del cual el crecimiento no se encuentra limitado, en condiciones de adecuada provisión de agua y otros nutrientes (Díaz-Zorita, 2002). 
Merrien (1992) indica que la concentración de $\mathrm{N}$ en la planta que permite alcanzar la máxima tasa de crecimiento se denomina concentración crítica, la que varía en función de la biomasa, ya que en la medida que ésta aumenta, la proporción de este macronutriente disminuye por efecto de su "dilución" en la biomasa aérea incrementada. Un indicador útil para la detección de deficiencias tempranas de $\mathrm{N}$, es el Índice de Nutrición Nitrogenada (INN) (Díaz-Zorita, 2002), que se calcula como el cociente entre la concentración de $\mathrm{N}$ en el cultivo $\mathrm{y}$ la concentración crítica estimada a partir de la materia seca, según la curva de regresión sugerida por Merrien (1992). En la medida en que el INN tienda a la unidad, la respuesta al agregado de $\mathrm{N}$ disminuye, independientemente de la dosis aplicada. Una metodología alternativa para la detección de deficiencias nitrogenadas en girasol, es el empleo de la curva de dilución del N (Greenwood et al., 1990), que determina para distintas acumulaciones de biomasa aérea, la concentración crítica de $\mathrm{N}$ que permite alcanzar al cultivo la máxima tasa de crecimiento (Díaz-Zorita y Duarte, 1998). Estas curvas pueden llegar a ser únicas para diferentes genotipos de una especie y hasta para un grupo de especies, y serían válidas para diferentes ambientes en condiciones de buena disponibilidad de agua (González Montaner et al., 1995; Andrade, 1995; DíazZorita y Duarte, 1998; Uhart y Echeverría, 2000).

Sosa et al. (1998) empleando la curva de Merrien (1992) en un ensayo de girasol en Balcarce, Argentina, encontraron que todos los valores calculados de nitrógeno foliar que se ubicaron por encima de la curva, indicaban que las plantas de girasol poseían un porcentaje de nitrógeno superior al necesario para lograr la máxima tasa de crecimiento del cultivo. Esta ecuación fue validada por varios investigadores (Andrade et al., 1995; Díaz-Zorita y Duarte, 1998; Uhart y Echeverría, 2000). Con concentraciones de $\mathrm{N}$ promedio de planta entera superiores a 3,5\% en etapa de 6-8 hojas, no se obtendrá respuesta al agregar este nutriente (González Montaner et al., 1995).

En el presente trabajo se planteó la hipótesis que un Argiustol Údico de la localidad de Presidencia Roque Sáenz Peña, Chaco, Argentina, laboreado en forma convencional ofrece una disponibilidad de nitrógeno suficiente para que el cultivo de Helianthus annuus se desarrolle normalmente. El objetivo del trabajo fue caracterizar el estado nutricional de nitrógeno en la biomasa aérea de dos híbridos de girasol, en distintas fases fenológicas, empleando la curva de dilución del nitrógeno en la materia seca aérea total.

\section{MATERIALES Y MÉTODOS}

El ensayo se realizó durante la campaña agrícola 2003-2004, en el campo experimental de la Estación Experimental Agropecuaria del Instituto Nacional de Tecnología Agropecuaria (INTA) de la localidad de Presidencia Roque Sáenz Peña, Chaco, Argentina ( $26^{\circ} 52^{\prime}$ lat. Sur; $60^{\circ} 27^{\prime}$ long. Oeste), sobre un Argiustol Údico, de la Serie Matanza, familia arcillosa fina, illítica, hipertérmica (Ledesma y Zurita, 1995).

Trabajo de campo. Se realizó un muestreo de suelo compuesto por 20 submuestras a $0-20 \mathrm{~cm}$ de profundidad, determinándose la siguiente composición: arena $=22 \%$; limo $=41 \%$; arcilla $=37 \%$; pH en agua $(1: 2,5)=6,88$; nitrógeno total por semi-microKjeldahl (Page et al., 1982) = 0,20\%; fósforo disponible $($ Bray, Kurtz I $)=81 \mathrm{mg} \mathrm{kg}^{-1}$ y materia orgánica (Walkley Black) = 2,52\%.

Los tratamientos fueron dos híbridos de girasol, GUARANI DC (Semillero Don Atilio) y DKSOL 4040 (Semillero Dekalb Argentina S.A.), que se seleccionaron por ser de ciclo largo y muy difundidos en la zona girasolera de la Provincia del Chaco. La preparación de la cama de siembra fue de tipo convencional, con dos pasadas de rastra de discos y dos pasadas cruzadas de cincel. El ensayo se sembró el 12 de agosto de 2003, con una densidad de 63.000 plantas $\mathrm{ha}^{-1}$, en surcos de $5,10 \mathrm{~m}$ de largo espaciados a $0,70 \mathrm{~m}$ y a razón de cuatro surcos por parcela. Se utilizó un diseño en bloques completamente aleatorizados con tres repeticiones por cada híbrido ensayado. Las malezas se controlaron químicamente con herbicidas y en forma manual hasta el final del ciclo de cultivo, y no se realizaron fertilizaciones por considerarse que el suelo no presentaba limitaciones nutricionales. No se realizaron controles fitosanitarios por no detectarse plagas y enfermedades que pudieran comprometer los resultados del ensayo. Se llevó un registro de precipitaciones y temperaturas durante el ciclo del cultivo (Cuadro 1).

Para definir las fases fenológicas del girasol se empleó la escala propuesta por Schneiter y Miller 
Cuadro 1. Registro de precipitaciones y temperaturas de Presidencia Roque Sáenz Peña, Chaco, Argentina. 2003. Table 1. Precipitation and temperature records in Presidencia Roque Sáenz Peña, Chaco, Argentina. 2003.

\begin{tabular}{lccccccccccccc}
\hline & En. & Feb. & Mar. & Ab. & May. & Jun. & Jul. & Ag. & Sept. & Oct. & Nov. & Dic. & Total \\
\hline Temp., ${ }^{\circ} \mathrm{C}$ & 26,6 & 25,3 & 23,9 & 20,8 & 17,8 & 19,5 & 15,5 & 14,8 & 20,3 & 13,5 & 24,5 & 24,8 & 20,61 \\
Prec., mm & 162,1 & 62,4 & 110,8 & 74,4 & 1,2 & 6,7 & 0 & 61,0 & 19,5 & 84,8 & 77,5 & 61,1 & 721,5 \\
\hline
\end{tabular}

(1981), quienes desarrollaron una escala macroscópica que permite identificar las fases y los estados macroscópicos relevantes del ciclo del girasol, registrándose también la altura de las plantas para cada una de las fases observadas. Los estadios fenológicos fueron: V6 = estado vegetativo con seis hojas verdaderas mayores de $4 \mathrm{~cm}$ de longitud; V10 $=$ estado vegetativo con 10 hojas verdaderas mayores de $4 \mathrm{~cm}$ de longitud; $\mathrm{R} 1$ = estado reproductivo que se alcanza cuando la inflorescencia, rodeada de brácteas inmaduras, se hace visible; y R5.5 = la antesis (R5) avanza en sentido centrípeto en el capítulo; y R6 que indica el fin de la floración. Se muestrearon tres plantas enteras de cada tratamiento a $\operatorname{los} 27,41,55,86$, y 106 días desde la siembra, correspondientes a cada uno de los estadios fenológicos mencionados.

Trabajo de laboratorio. Las plantas muestreadas se secaron en estufa a $70^{\circ} \mathrm{C}$, hasta peso constante, para determinar su biomasa aérea total para cada tratamiento y fase fenológica, posteriormente se pesaron y promediaron los valores de materia seca obtenidos en cada muestreo y fecha.

Para la determinación de la concentración del nitrógeno total de la biomasa aérea, se molieron las tres plantas correspondientes a cada tratamiento y fecha de muestreo, constituyendo una sola muestra a los fines de homogeneizar el material vegetal a analizar, determinando el nitrógeno total de cada híbrido y para cada fase fenológica muestreada. Merrien (1992) estableció la ecuación (A): \% $\mathrm{N}=$ 281,5 / (MS + 52,6), donde MS representa la materia seca aérea acumulada durante la fase vegetativa del cultivo. La determinación de nitrógeno foliar (B) se obtuvo en laboratorio por semi-microKjeldahl (Page et al., 1982). El análisis estadístico realizado fue la determinación del coeficiente de correlación y de determinación (Cuadro 2).

Cuadro 2. Principales resultados y coeficientes de correlación y determinación de nitrógeno (\%) de la ecuación (A) Merrien (1992) y nitrógeno foliar (B) en girasol.

Table 2. Main results and correlation coefficient and nitrogen (\%) determination of Merrien (1992) equation (A) and foliar nitrogen (B) in sunflower.

\begin{tabular}{|c|c|c|c|c|c|c|c|c|c|}
\hline Híbrido & $\begin{array}{c}\text { Días } \\
\text { desde } \\
\text { emergencia }\end{array}$ & $\begin{array}{c}\text { Fase } \\
\text { fenológica }\end{array}$ & $\begin{array}{c}\text { MS Total } \\
\left.\text { (g planta }^{-1}\right)\end{array}$ & $\begin{array}{r}\mathbf{N} \% \\
\text { (A) }\end{array}$ & $\begin{array}{c}\text { N\% } \\
\text { (B) }\end{array}$ & $\begin{array}{c}r \\
n=45\end{array}$ & $\mathbf{R}^{2}$ & $\begin{array}{l}\text { Rdmto. } \\
\left.\text { (kg ha' }{ }^{-1}\right)\end{array}$ & $\begin{array}{c}\text { Materia } \\
\text { grasa } \\
(\%)\end{array}$ \\
\hline & 10 & Emergencia & 0,40 & 5,31 & 4,21 & & & & \\
\hline & 27 & V 6 & 1,13 & 5,24 & 5,78 & & & & \\
\hline GUARANÍ & 41 & V 10 & 18,3 & 3,97 & 5,17 & $0,86^{* *}$ & 0,70 & 2.571 & 49,2 \\
\hline \multirow[t]{6}{*}{$\mathrm{DC}$} & 55 & R 1 & 30,5 & 3,39 & 3,06 & & & & \\
\hline & 86 & R 5.5 & 108,8 & 1,74 & 1,59 & & & & \\
\hline & 106 & R 6 & 226,0 & 1,01 & 0,99 & & & & \\
\hline & 10 & Emergencia & 0,40 & 5,31 & 4,21 & & & & \\
\hline & 27 & V 6 & 1,0 & 5,25 & 5,33 & & & & \\
\hline & 41 & V 10 & 18,4 & 3,97 & 5,27 & & & & \\
\hline DKSOL & 55 & R 1 & 21,2 & 3,81 & 3,17 & $0,82^{* *}$ & 0,67 & 2.571 & 46,2 \\
\hline \multirow[t]{2}{*}{4040} & 86 & R 5.5 & 74,4 & 2,22 & 2,67 & & & & \\
\hline & 106 & R 6 & 147,8 & 1,40 & 1,39 & & & & \\
\hline
\end{tabular}

MS: materia seca; N (\%) (A): nitrógeno según modelo de Merrien (1992); N (\%) (B): nitrógeno foliar semi-microKjeldahl; r: coeficiente de correlación; $\mathrm{R}^{2}$ : coeficiente de determinación.

$* *(\mathrm{p}<0,01)$. 


\section{RESULTADOS Y DISCUSIÓN}

El suelo es de textura franco-arcillosa, las concentraciones de nitrógeno y fósforo fueron consideradas adecuadas a altas. El pH y porcentaje de materia orgánica edáfica arrojaron valores normales para la zona, considerando que el suelo no presentaba limitaciones nutricionales para el cultivo de girasol. Las precipitaciones de enero a diciembre fueron de $721,5 \mathrm{~mm}$ y las temperaturas medias de $20,61^{\circ} \mathrm{C}$ (Cuadro 1$)$.

Para el híbrido GUARANI DC, en la fase fenológica V6 el contenido de nitrógeno aplicando la ecuación (A) fue 5,24\% y el nitrógeno foliar (B) fue $5,78 \%$ (Cuadro 2). Resultados similares se detectaron en la fase V10, encontrándose que el nitrógeno (A) fue 3,97\%, y el nitrógeno (B) fue $5,17 \%$, interpretándose que durante las fases vegetativas del girasol, el contenido de nitrógeno en las plantas es superior a la máxima tasa de crecimiento del cultivo (Aguirrezábal et al., 1996). A partir del comienzo de las fases reproductivas se observa que en $\mathrm{R} 1$ el nitrógeno $(\mathrm{A})=3,39 \%$ fue superior al del nitrógeno $(\mathrm{B})=3,06 \%$. Lo mismo sucede en la fase R5.5, en la cual el nitrógeno $(A)=1,74 \%$ es superior al nitrógeno $(\mathrm{B})=1,59 \%$, y en la fase R6 el nitrógeno $(\mathrm{A})=1,01 \%$ con valores similares al del nitrógeno $(\mathrm{B})=0,99 \%$, probablemente como consecuencia del aumento en las precipitaciones pluviales. Estos resultados se atribuyen al cambio en la composición de la materia seca aérea de las plantas, dando comienzo a la etapa de la síntesis de aceites (Merrien 1992; Connor y Sadrás, 1992; Satorre et al., 2004). En todos los casos se obtuvieron valores de correlación altamente significativos $(\mathrm{r}=$ $0,86)$ entre los métodos (A) y (B) para las distintas fases fenológicas muestreadas $(p<0,01)$ y con un promedio de materia grasa del $49,2 \%$.

Para el híbrido DKSOL 4040 en la fase fenológica V6, el contenido de nitrógeno aplicando la ecuación $(\mathrm{A})=5,25 \%$ y contenidos superiores de nitrógeno foliar (B) $=5,33 \%$ (Cuadro 2). Similares resultados se hallaron para la fase V10, donde el nitrógeno (A) fue 3,97\% y el nitrógeno (B) fue 5,27\%, interpretándose que en los estados de desarrollo vegetativo el cultivo no se encuentra limitado en este nutriente (Díaz-Zorita, 2002). Al comienzo de la fase reproductiva en R1, el porcentaje de nitrógeno (A) $(3,81 \%)$ es superior al del nitrógeno (B) $(3,17 \%)$, atribuyéndose al cambio en la composi- ción de la materia seca como consecuencia del comienzo de la fase reproductiva del cultivo. En la fase R5.5 el nitrógeno (A) fue 2,22\% y el nitrógeno (B) fue $2,67 \%$. Estos resultados se atribuyen a que existe una estrecha relación entre el nitrógeno en la planta entera y los niveles en el suelo en el momento de su medición (Díaz-Zorita y Duarte, 1998), y que éste haya aumentado probablemente como consecuencia de las mayores precipitaciones pluviales registradas durante la fase R5.5. En la fase R6 no se detectaron diferencias en los contenidos de nitrógeno en (A) $(1,40 \%)$ con el nitrógeno (B) $(1,39 \%)$. Los valores de correlación fueron altamente significativos $(\mathrm{r}=0,82)$ para ambos métodos $(\mathrm{A})$ y (B) y en las diferentes fases fenológicas muestreadas $(\mathrm{p}<0,01)$, arrojando un porcentaje promedio de materia grasa de $46,2 \%$.

En ambos híbridos estudiados los porcentajes de nitrógeno obtenidos en las fases de desarrollo estuvieron por encima de la curva de dilución del nitrógeno (Figuras 1 y 2), obteniéndose valores más erráticos cuando se inician los estadios reproductivos, manifestándose un déficit de nitrógeno que no sería utilizable para juzgar el estado nitrogenado del cultivo en estadios posteriores a la floración (Aguirrezábal et al.,1996), puesto que luego de dicha fase, cambia la composición de la materia seca, al comenzar la síntesis de aceite en los frutos. Además debe considerarse, que las curvas de dilución pueden llegar a ser únicas para diferentes genotipos de una especie y hasta para un grupo de espe-

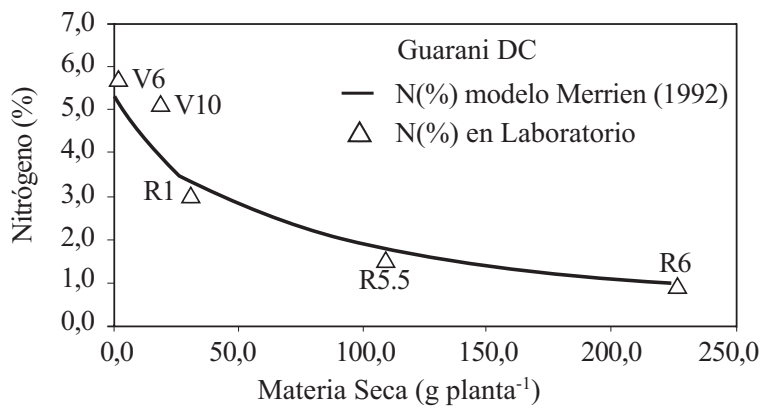

Figura 1. Porcentaje de nitrógeno en el híbrido de girasol GUARANI DC según la ecuación (A) de Merrien (1992) y determinación semi-micro Kjeldahl laboratorio (B).

Figure 1. Nitrogen percentage in GUARANI DC sunflower hybrid according to the Merrien (1992) equation (A) and by the semi-microKjeldahl $\operatorname{method}(B)$. 


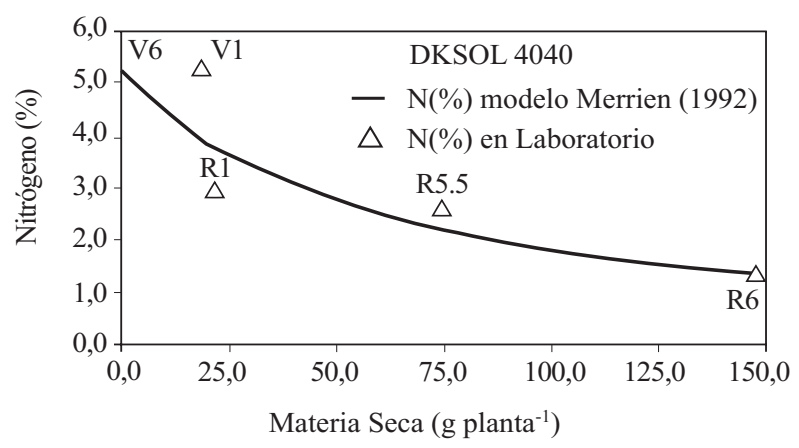

Figura 2 . Porcentaje de nitrógeno en el híbrido de girasol DKSOL 4040 según la ecuación (A) de Merrien (1992) y determinado en semi-micro Kjeldahl laboratorio (B).

Figure 2. Nitrogen percentage in DKSOL 4040 sunflower hybrid according to the Merrien equation (1992) (A) and by the semi-microKjeldahl method (B). cies, y serían válidas para diferentes ambientes en condiciones de buena disponibilidad de agua y nutrientes (Uhart y Echeverría, 2000). Con el cálculo del coeficiente de determinación se obtuvo para GUARANI DC $=0,70$ y DKSOL $4040=0,67$ (Cuadro 2), que indica la variabilidad del nitrógeno determinado en el laboratorio (B) con respecto al nitrógeno calculado en el modelo propuesto por Merrien (1992).

\section{CONCLUSIONES}

La utilización de la curva de dilución del nitrógeno permitió estimar el nivel nitrogenado del cultivo de Helianthus annuus, detectando que el contenido de nitrógeno en las plantas fue superior a la cantidad necesaria para la máxima tasa de crecimiento del cultivo durante los estadios vegetativos.

En la etapa reproductiva se detectó una disminución en la concentración de nitrógeno en planta, como consecuencia del aumento de la biomasa y de la traslocación de este nutriente de las hojas a los frutos.

\section{LITERATURA CITADA}

Aguirrezábal, L.A., G.A. Orioli, L.F. Hernández, V.R. Pereyra, y J.P. Miravé. 1996. Girasol. Aspectos fisiológicos que determinan el rendimiento. $127 \mathrm{p}$. Unidad Integrada Balcarce, Buenos Aires, Argentina.

Andrade, F.H. 1995. Analysis of growth and yield of maize, sunflower and soybean grown at Balcarce, Argentina. Field Crops Res. 41:1-12.

Andrade, F. H., L.A. Aguirrezábal, y R.H. Rizzalli. 2002. Crecimiento y rendimiento comparados. 57-96. In Andrade F.H., y Sadras V.O. (eds.). Bases para el manejo del maíz, el girasol y la soja. $2^{\mathrm{a}}$ ed. E.E.A. INTA Balcarce - Facultad de Ciencias Agrarias Universidad Nacional de Mar del Plata, Balcarce, Argentina.

Connor, D.J., and V.O. Sadras. 1992. Physiology of yield expression in sunflower. Field Crop Res. 30:333-389.

Díaz-Zorita, M. 1997. Manejo del agua y de la nutrición mineral. Curso de actualización "Manejo del cultivo de girasol". p. 79-94. INTA, E.E.A. Gral. Villegas, Argentina.

Díaz-Zorita, M. 2002. Nutrición mineral y fertilización. p. 77-96. In Díaz-Zorita, M., y Duarte, G.A. Manual práctico para el cultivo de girasol. Editorial Hemisferio Sur-INTA, Buenos Aires, Argentina.
Díaz-Zorita, M., y G. Duarte. 1998. El nitrógeno y la producción de girasol en la región de la pampa arenosa. p.115-116. In XVI Congreso Argentino de la Ciencia del Suelo, Carlos Paz, Córdoba. Asociación Argentina de la Ciencia del Suelo, Argentina.

González Montaner, J., M. Posborg, F. Dolorico, y W. Wagner. 1995. Girasol. Diagnóstico de fertilización nitrogenada en el sudeste de Buenos Aires. Revista CREA N ${ }^{\circ} 181$ p. 52-57.

Greenwood, D.J., G. Lemaire, G. Grosse, P. Cruz, A. Draycott, and J. Neeteson.1990. Decline in percentage $\mathrm{N}$ of $\mathrm{C} 3$ and $\mathrm{C} 4$ crops with increasing plant mass. Annals Bot. London. 66: 425-436.

Hocking, P.J., and B.T. Steer. 1983. Uptake and partitioning of selected mineral elements in sunflower (Helianthus annuus L.) during growth. Field Crop Res. 6:93-107.

Ledesma, L.L., y J.J. Zurita. 1995. Los suelos de la Provincia del Chaco. 164 p. Convenio Instituto Nacional de Tecnología Agropecuaria (INTA)/ Gobierno de la Provincia del Chaco. Ministerio de Agricultura y Ganadería, Chaco, Argentina. 
Loomis, R.S., D.J. Connor. 2002. Ecología de cultivos. Productividad y manejo en sistemas agrarios. $591 \mathrm{p}$. Ediciones Mundi-Prensa, Madrid, España.

Merrien, A. 1992. Physiologie du tournesol. 65 p. Centre Technique Interprofessionnel des Oléagineux Métropolitain (CETIOM), Paris, France.

Page, A.L., R.H. Miller, and D.R. Keeney. 1982. Methods of soil analysis. 1.159 p. Part 2. Chemical and microbiological properties. $2^{\text {nd }}$ ed. American Society of Agronomy, Madison, Wisconsin, USA.

Ruffo M.L., F.O García, G.A. Bollero, K. Fabrizzi, and R.A. Ruiz. 2003. Nitrogen balance approach to sunflower fertilization. Commun. Soil Sci Plant Anal. 34:2645-2657.

Sadrás, V.O., M. Ferreiro, F. Gutheim, y A.G. Kantolic. 2002. Desarrollo fenológico y su respuesta a temperatura y fotoperíodo. p. 25-56. In Andrade F.H., y V.O. Sadrás (eds.). Bases para el manejo del maíz, el girasol y la soja. $2^{a}$ ed. INTA-E.E.A. Balcarce Universidad Nacional de Mar del Plata, Facultad de Ciencias Agrarias, Balcarce, Argentina.
Satorre, E.H., R.L. Benech Arnold, G.A. Slafer, E.B. de la Fuente, D.J. Miralles, M.E. Otegui, y R. Savin. 2004. Producción de granos. Bases funcionales para su manejo. 783 p. Universidad de Buenos Aires, Facultad de Agronomía, Buenos Aires, Argentina.

Sosa, L., G. Dosio, L.A. Aguirrezábal, y H.E. Echeverría. 1998. Evaluación de la nutrición nitrogenada del girasol cultivado en Balcarce mediante la curva de dilución. p. 121-122. In Actas III Reunión Nacional de Oleaginosas, Bahía Blanca. Mayo de 1998. Asociación Argentina de Oleaginosas, Buenos Aires, Argentina.

Schneiter, A., and J.F. Miller. 1981. Description of sunflower growth stages. Crop Sci. 21:901-903.

Uhart, S.A., y H.E. Echeverría. 2000. Diagnóstico de la fertilización. p. 239-272. In Andrade F.H., y V.O. Sadrás (eds.). Bases para el manejo del maíz, el girasol y la soja. $2^{\mathrm{a}}$ ed. INTA E.E.A. Balcarce - Universidad Nacional de Mar del Plata, Facultad de Ciencias Agrarias, Balcarce, Argentina. 